\title{
Role of RNA-Binding Proteins in Colorectal Carcinogenesis
}

\author{
Shrikant Anant, \\ Section of Digestive Diseases and Nutrition, Department of Internal Medicine, University of \\ Oklahoma Health Sciences Center, 920 Stanton L. Young Boulevard WP1345, Oklahoma City, \\ OK 73104, USA; Cell Biology, University of Oklahoma Health Sciences Center, Oklahoma City, \\ OK 73104, USA \\ Courtney W. Houchen, \\ Section of Digestive Diseases and Nutrition, Department of Internal Medicine, University of \\ Oklahoma Health Sciences Center, 920 Stanton L. Young Boulevard WP1345, Oklahoma City, \\ OK 73104, USA \\ Vaibhav Pawar, and \\ Section of Digestive Diseases and Nutrition, Department of Internal Medicine, University of \\ Oklahoma Health Sciences Center, 920 Stanton L. Young Boulevard WP1345, Oklahoma City, \\ OK 73104, USA

\section{Satish Ramalingam} \\ Section of Digestive Diseases and Nutrition, Department of Internal Medicine, University of \\ Oklahoma Health Sciences Center, 920 Stanton L. Young Boulevard WP1345, Oklahoma City, \\ OK 73104, USA
}

\section{Abstract \\ RNA-binding proteins (RBPs) play key roles in the posttranscriptional regulation of gene expression. RBPs control various posttranscriptional events, including splicing, polyadenylation, mRNA stability, transport, and translation. It is becoming apparent that RBPs play a significant role in pathophysiologic conditions such as inflammation and cancer. More importantly, we and others have begun dissecting the role of mRNA stability and translation in regulating gene expression, dysregulation of which has serious consequences for the fate of the cell. In this article, we discuss this emerging area of posttranscriptional gene regulation and the role of RBPs in the aberrant expression of proteins in tumorigenesis.}

\section{Keywords}

Colon cancer; Gene regulation; Posttranscriptional regulation; RNA stability; mRNA translation; mRNA binding proteins; Protooncogenes; Protein:nucleic acid interactions; Protein:protein interactions; Cyclooxygenase-2; HuR; Tristetraprolin; CUGBP2; Musashi-1

\section{Introduction}

RNA-binding proteins (RBPs) are key components in RNA metabolism that regulate all aspects of RNA biogenesis, including RNA splicing; polyadenylation; nucleocytoplasmic transport, resulting in highly organized subcellular localization; and finally, but not the least

(C) Springer Science+Business Media, LLC 2010

shrikant-anant@ouhsc.edu .

Disclosure No potential conflicts of interest relevant to this article were reported. 
important, mRNA translation and RNA degradation [1-3]. The RBPs have an organized structure in which they interact with one another in solution and form dynamic interactions with coding, untranslated, and non-protein-coding RNAs in functional units called ribonucleoprotein (RNP) complexes [4]. The RBPs then modulate the function of the RNP complex in a temporal and spatial manner. The best-characterized RNP complexes to date are those that interact at the exon junction complex to regulate splicing $[5,6]$. These complexes have been identified to contain upward of 10 proteins, and some remain bound until the RNA is translated in the cytoplasm.

RNA stability and translation controls have lagged behind our understanding of gene regulation, the beststudied aspect being ribosome assembly and processing. The ribosome is composed of a small (40 S) and a large (60 S) subunit as well as several ribosomal RNA molecules and proteins (Fig. 1). Ribosome-mediated translation occurs in three stages: initiation, elongation, and termination. Initiation occurs with the $40 \mathrm{~S}$ subunit attaching to the mRNA and scanning for the start AUG codon that encodes for methionine [7,8]. Once the AUG is identified, the $60 \mathrm{~S}$ subunit binds and elongation begins. Finally, termination occurs when the ribosome reaches a stop codon. The new protein is then released, and the translation complex comes apart. Based on the current hypothesis, this translation machinery continues in the form of an assembly-line apparatus, with termination occurring upon the initiation of degradation through reduction of the poly(A) tail at the $3^{\prime}$ end of the mRNA (Fig. 1).

Currently, the exciting area of investigation in the control of mRNA translation is in identifying and characterizing cellular proteins that bind the mRNA and regulate translation. Key elements that have received much attention are the AU-rich element (ARE) sequences generally located within the $3^{\prime}$ untranslated regions ( $3^{\prime}$ UTRs) of the mRNA. The first factor to be identified, and the one that remains a "gold standard" in the area of mRNA stability and translation, is the $\mathrm{Hu}$ antigen $\mathrm{R}(\mathrm{HuR})$ protein $[9,10]$. More recently, additional proteins have been identified that can bind independently to the mRNAs or complex with HuR to regulate translational activity [11].

\section{HuR}

The HuR protein is a ubiquitous member of the $E L A V$-like family of RBPs. It consists of 326 amino acids, resulting in a molecular mass of $36 \mathrm{kD}$, and encodes three RNArecognition motifs (RRMs) through which it binds with high affinity to specific mRNAs bearing AU-and U-rich sequences, affecting their stability and translation. Increased HuR expression has been observed in multiple cancers, including colorectal tumors [11-13]. However, in many cases, it is not the expression of the protein per se that correlates with tumorigenesis, but rather the subcellular localization with higher cytoplasmic levels observed in cancers. When overexpressed, HuR stabilizes ARE-containing transcripts and promotes their translation [14]. In colorectal cancers, HuR has been shown to increase the stability and translation of cyclooxygenase $2(\mathrm{COX}-2)$ mRNA by binding to ARE sequences located within the $3^{\prime}$ UTR immediately downstream of the coding region $[11,15,16]$. Changes in HuR expression were found to occur early during tumorigenesis, and this alteration was associated with the increased COX-2 levels observed in tumors. In normal cells, HuR is localized mainly in the nucleus. Export of HuR to the cytoplasm is associated with ARE-containing mRNA stabilization [14]. Increased cytoplasmic localization of HuR contributes to COX-2 overexpression in colon cancer cells [9]. However, recent evidence suggests that the context of HuR interactions with the mRNA is essential for HuR-mediated function. If the protein interacts with factors such as RBM3, then it will be a positive translation regulator, whereas interaction with CUGBP2 or tristetraprolin (TTP) would result in translation inhibition (Fig. 1). The control of COX-2 expression is a complex regulatory 
process that requires input from multiple pathways, which affect gene expression on both transcriptional and posttranscriptional levels. It is well known that transcriptional activation of the COX-2 gene is an early event in tumorigenesis because evidence demonstrates the presence of COX-2 mRNA in all colorectal adenomas, adenocarcinomas, and colon cancer cells $[17,18]$. Although there is clear evidence that HuR regulates COX-2 expression in colon cancer development, the exact role the protein plays in inducing oncogenic events is not yet known. One important point that must be determined further is whether HuR is a protooncogene that, when overexpressed, would transform a normal cell. If HuR was not found to transform cells, then it might play a role in disease progression. Knowing whether HuR can transform a cell or is involved in tumor progression will help in developing inhibitors for the protein. In this regard, we recently demonstrated that RBP RBM3 is a proto-oncogene.

\section{RBM3}

In a yeast two-hybrid screening analysis using HuR as bait, we identified RNA binding motif protein RBM3 as a binding partner. The RBM3 gene, first identified from the complementary DNA library of human fetal brain tissue, encodes two alternatively spliced RNA transcripts [19]. The longest open reading frame has 157 amino acids with a molecular mass of $17 \mathrm{kD}$ and consists of one RRM domain and a glycine-rich region. RBM3 regulates global mRNA translation by interacting with the $60 \mathrm{~S}$ ribosome [19-21]. Like HuR, RBM3 is significantly upregulated in human tumors, with a stepwise, stage-dependent increase observed in colorectal tumors. Moreover, although RBM3 is confined mostly to the nucleus in normal cells, it was observed to also be cytoplasmic, especially in the later stages of tumorigenesis [22•]. Overexpression of RBM3 in NIH3T3 mouse fibroblasts resulted in the development of compact multicellular spheroids in soft agar, suggesting this protein's ability to induce anchorage-independent growth, a characteristic of transformed cells [22•]. In contrast, when RBM3 expression was downregulated in HCT116 colon cancer cells using a specific silencer RNA, there was a reduction in cell growth in culture and in the growth of tumor xenografts. Moreover, there was a significant reduction in tumor angiogenesis in the xenografts, determined by reduction in capillary formation. This was determined to be the result of a reduction in the expression of proangiogenic factors COX-2, vascular endothelial growth factor (VEGF), and interleukin-8 (IL-8) [22]. Another key aspect of RBM3 that was observed is that it protects the cells from mitotic catastrophe. When RBM3 was downregulated, there was an increase in $\mathrm{Cdc} 25 \mathrm{c}$ protein levels. Cdc25c catalyzes the activation of the $\mathrm{Cdk}$-cyclin $\mathrm{B} 1$ kinase complex, which is believed to be a rate-limiting step for entry into mitosis [23,24]. Indeed, the cells had high levels of phosphorylated histone H3, a hallmark for cells in mitosis. At the same time, the RBM3-depleted cells underwent apoptosis as evidenced by the increased levels of TUNEL positivity. Thus, these data clearly demonstrate a critical role for the ubiquitously expressed RBM3 protein in protecting the cells during mitosis. Mechanistically, RBM3 is a nucleo-cytoplasmic shuttling protein that stabilizes COX-2, VEGF, and IL-8 mRNAs. RBM3 protein binds to the COX-2 ARE and regulates COX-2 mRNA stability and translation [20,22•]. Given that COX-2 is the ratelimiting enzyme in the production of prostaglandins, an important mediator of various cellular processes — such as proliferation, apoptosis resistance, and enhanced angiogenesisthat is overexpressed in multiple tumors [17,18], it is clear that RBM3 is central in the tumorigenesis process. RBM3 exerts its effects by increasing mRNA stability and translation of otherwise rapidly degraded transcripts. Hence, a thorough search for RBM3regulated transcripts will help in deciphering the role of RBM3 in tumorigenesis. 


\section{CUGBP2}

CUGBP2 (CUG triplet repeat-binding protein 2, also known as ETR3, NAPOR2, and BRUNOL2) is a ubiquitously expressed RBP of 490 amino acids and a molecular mass of $54 \mathrm{kD}$. It originally was identified in skeletal muscle through its interaction with CUG triplet repeats $[25,26]$. CUGBP2 belongs to a family of proteins collectively termed CELF (CUGBP1, CUGBP2, and CELF3 through CELF6) [27]. The members of this family contain two RRMs near the $\mathrm{NH}_{2}$ terminus and a third RRM near the $\mathrm{COOH}$ terminus $[26,28,29]$. CUGBP2 also was originally identified by a differential display analysis of neuroblastoma cells [30]. There are at least three variants identified for this protein, resulting from alternative promoter usage and alternative inclusion of the first exon. Differential expression of these variants has been identified in human colon cancer cells, suggesting a potential role for the variants in tumorigenesis [31•].

Mechanistically, CUGBP2 binds to AREs in the first 60 nucleotides of COX-2 3'UTR [32]. After binding, CUGBP2 increased the stability of COX-2 mRNA but inhibited translation. This is in contrast to HuR, which increased the stability and translation of COX-2 mRNA after binding to the ARE sequences. An added level of complexity was identified in that HuR and CUGBP2 can heterodimerize both in solution and on the RNA, suggesting that CUGBP2 modulates HuR function [33•]. A critical aspect of CUGBP2 is that its expression is relatively lower in colon cancer cells in culture than in normal epithelial cells. When the protein was overexpressed in HT-29 colon cancer cells, the cells underwent apoptotic cell death [32]. The mechanism behind this role is related to the loss of COX-2 protein expression, thereby decreasing prostaglandin $\mathrm{E}_{2}$ production. CUGBP2 also regulates COX-2 mRNA translation in response to $\gamma$-irradiation [34,35]. Subsequent studies determined that CUGBP2 overexpression results in cancer cells undergoing mitotic catastrophe $[31 \bullet, 34]$. When CUGBP2 was overexpressed in HCT-116 colon cancer cells, there was a significant increase in cells transiting the G2/M phase. Moreover, higher levels of Chk1, Chk2, and its target $\mathrm{Cdc} 25 \mathrm{c}$ protein, coupled with higher levels of p53 phosphorylation (Ser15), were observed in the CUGBP2-expressing cells. In addition, both cyclin B1 and Cdc2 were observed to be in the nucleus, and cells had higher levels of phosphorylated histone H3 [31•, 36]. This suggests that when CUGBP 2 is overexpressed, the cells are driven toward mitosis through the actions of Chk1/Chk2 kinases while the cells undergo apoptosis driven by activated p53. Further studies are needed to determine the role of the different variants of CUGBP2 in tumorigenesis, especially because only one of the three variants could induce mitotic catastrophe.

\section{Musashi-1}

Musashi-1 (Msi1), originally identified in Drosophila, is required for the asymmetric cell division of sensory neural precursor cells [37]. Msi1 protein consists of 362 amino acids with a predicted molecular mass of $39 \mathrm{kD}$ and contains two conserved RRMs in its Nterminus. It has been observed to be localized predominantly in the cytoplasm in embryonic neural progenitor cells, which may be explained by the presence of a putative nuclear export signal in its C-terminus [38]. Msi1 has been shown to be a positive regulator of Notch signaling through its interaction and translational repression of mammalian Numb (mNumb mRNA [an inhibitor of Notch signaling]) [39]. Another transcript known to be a target for Msi1 is the mRNA that encodes p21WAF1 [40]. However, unlike CUGBP2, Msi1 does not affect the steady-state levels of the mRNA. Further studies are required to further define the mechanism by which Msi1 regulates mRNA translation, especially because it has been found to associate with monoribosomes and polyribosomes. Msi1 expression correlates with colon carcinogenesis [41]. In a recent study, Msi1 protein expression was significantly higher in stage III than stage I and II colon cancer tissue samples, suggesting that Msi1 may 
be involved in tumor invasion and metastasis. Furthermore, Msi1 knockdown resulted in tumor growth arrest in xenografts, reduced cancer cell proliferation, and increased apoptosis, alone and in combination with radiation injury [42]. These studies suggest that targeting Msi1, both by itself and as a radiosensitizer, might be an attractive target for cancer therapy.

TTP

TTP (also called ZFP36, TIS11) is a member of a small family of tandem Cys3His zinc finger proteins that originally was identified as a tumor necrosis factor (TNF)- $\alpha$ AREbinding protein [43]. Knockout of TTP results in increased stabilization of TNF- $\alpha$ mRNA in lipopolysaccharide-induced macrophages. TTP is a cytoplasmic protein that interacts with the exosome components to induce mRNA degradation [44]. TTP can inhibit tumorigenesis of an H-ras-dependent mast cell model, which occurs through degradation of IL-3 mRNA [45]. Loss of TTP is a critical factor for cancer-associated gene overexpression in tumors. Low levels of TTP are sufficient to efficiently suppress COX-2 expression in cells, even in the presence of elevated HuR [46••]. Thus, it is believed that like CUGBP2, TTP probably is a tumor suppressor that inhibits expression of genes that encode an ARE in the 3'UTR. However, the only difference between CUGBP2 and TTP is that TTP induces deadenylation whereas CUGBP2 does not. Further studies are required to determine how CUGBP2 inhibits translation. More importantly, given that both proteins interact with HuR, it remains to be determined whether the interactions of the two proteins with HuR are mutually exclusive or whether they can be in the same complex.

\section{Conclusions}

These are exciting times for researchers in the area of RBPs and tumorigenesis. We are only beginning to understand posttranscriptional regulation of mRNA stability and translation. Only a handful of proteins have been identified in the process (Fig. 1), but it is not too farfetched to think there are many proteins that remain to be discovered. Unlike in the past, there now are many innovative techniques that can be used to carefully isolate protein complexes on the RNA. Once these are isolated, powerful biochemical techniques, such as mass spectrophotometry analyses, can be used to identify the proteins in the complexes. Uncovering proteins in the complex subsequently will help dissect the pathways that regulate the expression and function of these proteins and perhaps also identify novel therapeutic targets for colorectal and other cancers.

\section{References}

Papers of particular interest, published recently, have been highlighted as:

- Of importance

•• Of major importance

1. Saunus JM, French JD, Edwards SL, et al. Posttranscriptional regulation of the breast cancer susceptibility gene BRCA1 by the RNA binding protein HuR. Cancer Res 2008;68:9469-9478. [PubMed: 19010922]

2. Shen L, Pili R. Posttranscription regulation of prostate cancer growth. Cancer J 2008;14:46-53. [PubMed: 18303483]

3. Lu Z, Luo RZ, Peng H, et al. Transcriptional and posttranscriptional down-regulation of the imprinted tumor suppressor gene ARHI [DRAS3] in ovarian cancer. Clin Cancer Res 2006;12:2404-2413. [PubMed: 16638845]

4. Ule J. Ribonucleoprotein complexes in neurologic diseases. Curr Opin Neurobiol 2008;18:516-523. [PubMed: 18929657] 
5. Le Hir H, Seraphin B. EJCs at the heart of translational control. Cell 2008;133:213-216. [PubMed: 18423193]

6. Tange TO, Shibuya T, Jurica MS, Moore MJ. Biochemical analysis of the EJC reveals two new factors and a stable tetrameric protein core. RNA 2005;11:1869-1883. [PubMed: 16314458]

7. Kamoshita N, Nomoto A, RajBhandary UL. Translation initiation from the ribosomal A site or the P site, dependent on the conformation of RNA pseudoknot I in dicistrovirus RNAs. Mol Cell 2009;35:181-190. [PubMed: 19647515]

8. Aliprandi P, Sizun C, Perez J, et al. S1 ribosomal protein functions in translation initiation and ribonuclease RegB activation are mediated by similar RNA-protein interactions: an NMR and SAXS analysis. J Biol Chem 2008;283:13289-13301. [PubMed: 18211890]

9. Brennan CM, Steitz JA. HuR and mRNA stability. Cell Mol Life Sci 2001;58:266-277. [PubMed: 11289308]

10. Doller A, Pfeilschifter J, Eberhardt W. Signaling pathways regulating nucleo-cytoplasmic shuttling of the mRNA-binding protein HuR. Cell Signal 2008;20:2165-2173. [PubMed: 18585896]

11. Dixon DA, Tolley ND, King PH, et al. Altered expression of the mRNA stability factor HuR promotes cyclooxygenase-2 expression in colon cancer cells. J Clin Invest 2001;108:1657-1665. [PubMed: 11733561]

12. de Silanes, I Lopez; Fan, J.; Yang, X., et al. Role of the RNA-binding protein HuR in colon carcinogenesis. Oncogene 2003;22:7146-7154. [PubMed: 14562043]

13. Denkert C, Koch I, von Keyserlingk N, et al. Expression of the ELAV-like protein HuR in human colon cancer: association with tumor stage and cyclooxygenase-2. Mod Pathol 2006;19:12611269. [PubMed: 16799479]

14. Fan XC, Steitz JA. Overexpression of HuR, a nuclear-cytoplasmic shuttling protein, increases the in vivo stability of ARE-containing mRNAs. EMBO J 1998;17:3448-3460. [PubMed: 9628880]

15. Subbaramaiah K, Marmo TP, Dixon DA, Dannenberg AJ. Regulation of cyclooxgenase-2 mRNA stability by taxanes: evidence for involvement of p38, MAPKAPK-2, and HuR. J Biol Chem 2003;278:37637-37647. [PubMed: 12826679]

16. Sawaoka H, Dixon DA, Oates JA, Boutaud O. Tristetraprolin binds to the 3'-untranslated region of cyclooxygenase-2 mRNA. A polyadenylation variant in a cancer cell line lacks the binding site. J Biol Chem 2003;278:13928-13935. [PubMed: 12578839]

17. Eberhart CE, Coffey RJ, Radhika A, et al. Up-regulation of cyclooxygenase 2 gene expression in human colorectal adenomas and adenocarcinomas. Gastroenterology 1994;107:1183-1188. [PubMed: 7926468]

18. Shao J, Sheng H, Inoue H, et al. Regulation of constitutive cyclooxygenase-2 expression in colon carcinoma cells. J Biol Chem 2000;275:33951-33956. [PubMed: 10930401]

19. Derry JM, Kerns JA, Francke U. RBM3, a novel human gene in Xp11.23 with a putative RNAbinding domain. Hum Mol Genet 1995;4:2307-2311. [PubMed: 8634703]

20. Danno S, Nishiyama H, Higashitsuji H, et al. Increased transcript level of RBM3, a member of the glycine-rich RNA-binding protein family, in human cells in response to cold stress. Biochem Biophys Res Commun 1997;236:804-807. [PubMed: 9245737]

21. Dresios J, Aschrafi A, Owens GC, et al. Cold stress-induced protein Rbm3 binds $60 \mathrm{~S}$ ribosomal subunits, alters microRNA levels, and enhances global protein synthesis. Proc Natl Acad Sci U S A 2005;102:1865-1870. [PubMed: 15684048]

22. Sureban SM, Ramalingam S, Natarajan G, et al. Translation regulatory factor RBM3 is a protooncogene that prevents mitotic catastrophe. Oncogene 2008;27:4544-4556. [PubMed: 18427544] This is the first demonstration that an RBP is a proto-oncogene.

23. Maller JL. Mitotic control. Curr Opin Cell Biol 1991;3:269-275. [PubMed: 1883620]

24. Millar JB, Russell P. The cdc25 M-phase inducer: an unconventional protein phosphatase. Cell 1992;68:407-410. [PubMed: 1310893]

25. Choi DK, Ito T, Tsukahara F, et al. Developmentally-regulated expression of mNapor encoding an apoptosis-induced ELAV-type RNA binding protein. Gene 1999;237:135-142. [PubMed: 10524244] 
26. Lichtner P, Attie-Bitach T, Schuffenhauer S, et al. Expression and mutation analysis of BRUNOL3, a candidate gene for heart and thymus developmental defects associated with partial monosomy 10p. J Mol Med 2002;80:431-442. [PubMed: 12110949]

27. Lu X, Timchenko NA, Timchenko LT. Cardiac elav-type RNA-binding protein [ETR-3] binds to RNA CUG repeats expanded in myotonic dystrophy. Hum Mol Genet 1999;8:53-60. [PubMed: 9887331]

28. Ladd AN, Nguyen NH, Malhotra K, Cooper TA. CELF6, a member of the CELF family of RNAbinding proteins, regulates muscle-specific splicing enhancer-dependent alternative splicing. J Biol Chem 2004;279:17756-17764. [PubMed: 14761971]

29. Ladd AN, Charlet N, Cooper TA. The CELF family of RNA binding proteins is implicated in cellspecific and developmentally regulated alternative splicing. Mol Cell Biol 2001;21:1285-1296. [PubMed: 11158314]

30. Choi DK, Ito T, Mitsui Y, Sakaki Y. Fluorescent differential display analysis of gene expression in apoptotic neuroblastoma cells. Gene 1998;223:21-31. [PubMed: 9858671]

31 . Ramalingam S, Natarajan G, Schafer C, et al. Novel intestinal splice variants of RNA-binding protein CUGBP2: isoform-specific effects on mitotic catastrophe. Am J Physiol Gastrointest Liver Physiol 2008;294:G971-G981. [PubMed: 18258790] This is the first demonstration of an RBP-regulating mitotic catastrophe.

32. Mukhopadhyay D, Houchen CW, Kennedy S, et al. Coupled mRNA stabilization and translational silencing of cyclooxygenase-2 by a novel RNA binding protein, CUGBP2. Mol Cell 2003;11:113126. [PubMed: 12535526]

33-. Sureban SM, Murmu N, Rodriguez P, et al. Functional antagonism between RNA binding proteins HuR and CUGBP2 determines the fate of COX-2 mRNA translation. Gastroenterology 2007;132:1055-1065. [PubMed: 17383427] This article demonstrates that the context of HuR binding to the COX-2 mRNA regulates translation function.

34. Natarajan G, Ramalingam S, Ramachandran I, et al. CUGBP2 downregulation by prostaglandin E2 protects colon cancer cells from radiation-induced mitotic catastrophe. Am J Physiol Gastrointest Liver Physiol 2008;294:G1235-G1244. [PubMed: 18325984]

35. Murmu N, Jung J, Mukhopadhyay D, et al. Dynamic antagonism between RNA-binding protein CUGBP2 and cyclooxygenase-2-mediated prostaglandin E2 in radiation damage. Proc Natl Acad Sci U S A 2004;101:13873-13878. [PubMed: 15358864]

36. Subramaniam D, Natarajan G, Ramalingam S, et al. Translation inhibition during cell cycle arrest and apoptosis: Mcl-1 is a novel target for RNA binding protein CUGBP2. Am J Physiol Gastrointest Liver Physiol 2008;294:G1025-1032. [PubMed: 18292181]

37. Nakamura M, Okano H, Blendy JA, Montell C. Musashi, a neural RNA-binding protein required for Drosophila adult external sensory organ development. Neuron 1994;13:67-81. [PubMed: 8043282]

38. Sakakibara S, Imai T, Hamaguchi K, et al. Mouse-Musashi-1, a neural RNA-binding protein highly enriched in the mammalian CNS stem cell. Dev Biol 1996;176:230-242. [PubMed: 8660864]

39. Okano H, Imai T, Okabe M. Musashi: a translational regulator of cell fate. J Cell Sci 2002;115:1355-1359. [PubMed: 11896183]

40. Battelli C, Nikopoulos GN, Mitchell JG, Verdi JM. The RNA-binding protein Musashi-1 regulates neural development through the translational repression of p21WAF-1. Mol Cell Neurosci 2006;31:85-96. [PubMed: 16214366]

41. Fan LF, Dong WG, Jiang CQ, et al. Expression of putative stem cell genes Musashi-1 and beta1integrin in human colorectal adenomas and adenocarcinomas. Int J Colorectal Dis 2010;25:17-23. [PubMed: 19714342]

42. Sureban SM, May R, George RJ, et al. Knockdown of RNA binding protein Musashi-1 leads to tumor regression in vivo. Gastroenterology 2008;134:1448-1458. [PubMed: 18471519]

43. Lai WS, Carballo E, Strum JR, et al. Evidence that tristetraprolin binds to AU-rich elements and promotes the deadenylation and destabilization of tumor necrosis factor alpha mRNA. Mol Cell Biol 1999;19:4311-4323. [PubMed: 10330172]

44. Hau HH, Walsh RJ, Ogilvie RL, et al. Tristetraprolin recruits functional mRNA decay complexes to ARE sequences. J Cell Biochem 2007;100:1477-1492. [PubMed: 17133347] 
45. Stoecklin G, Gross B, Ming XF, Moroni C. A novel mechanism of tumor suppression by destabilizing AU-rich growth factor mRNA. Oncogene 2003;22:3554-3561. [PubMed: 12789264]

46••. Young LE, Sanduja S, Bemis-Standoli K, et al. The mRNA binding proteins HuR and tristetraprolin regulate cyclooxygenase 2 expression during colon carcinogenesis. Gastroenterology 2009;136:1669-1679. [PubMed: 19208339] This landmark article demonstrates the differential roles of HuR and TTP in regulating COX-2 gene expression 


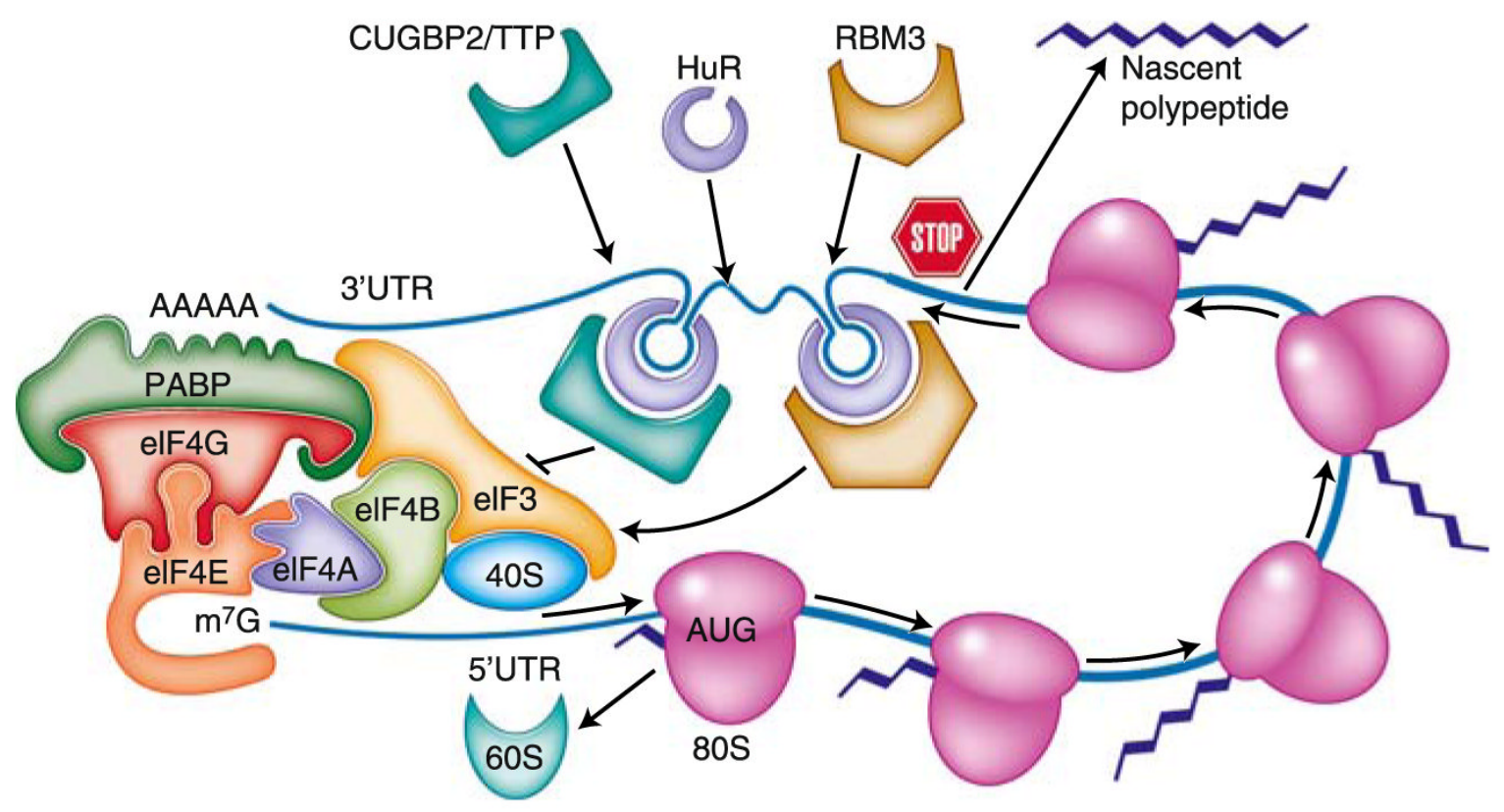

Fig. 1.

Classical protein translation and mechanisms of control involving RNA-binding proteins (RBPs). The important events in translation-initiation include the eukaryotic translation initiation factor $4 \mathrm{~F}$ (eIF4F) initiation complex, consisting of eIF4A, eIF4G, and eIF4E to the $\mathrm{m} 7 \mathrm{GpppN}$ cap, which is recruited and joined by eIF4B to form the $40 \mathrm{~S}$ ribosomal initiation complex. The $40 \mathrm{~S}$ ribosomal subunit complex scans across the $5^{\prime}$ untranslated region (5'UTR) to the AUG initiation codon, and the $60 \mathrm{~S}$ ribosomal subunit is recruited. During protein elongation, multiple $80 \mathrm{~S}$ ribosomes transit the open reading frame while elongating the newly synthesized peptide chain. During termination, the ribosome recognizes a stop codon and the $80 \mathrm{~S}$ ribosomal subunits dissociate from the mRNA. Also shown are RPBs RNA binding motif 3 (RBM3), Hu antigen R (HuR), CUG binding protein 2 (CUGBP2), and tristetraprolin (TTP), which can bind to stem-loop structures formed by AU-rich sequences in the $3^{\prime}$ untranslated region (3'UTR). HuR was the first RBP shown to bind to AU-rich elements in cyclooxygenase 2 mRNA and induce translation. Subsequent studies determined that RBPs CUGBP2 and TTP also bind to the mRNA but inhibit translation. More recent studies determined that the context of HuR interaction with its binding partners RBM3 and CUGBP2/TTP will determine whether the mRNA will be translated or not, respectively. mRNA and proteins are not drawn to scale. $\mathrm{PABP}-\mathrm{poly}(\mathrm{A})$ binding protein 